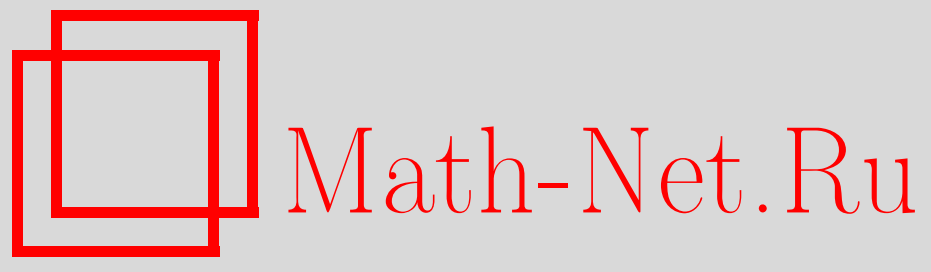

А. В. Устинов, Об одном обобщении сумм Клостермана, Матем. заметки, 2015, том 97, выпуск 1, 154-157

DOI: https://doi.org/10.4213/mzm10575

Использование Общероссийского математического портала Math-Net.Ru подразумевает, что вы прочитали и согласны с пользовательским соглашением http://www . mathnet.ru/rus/agreement

Параметры загрузки:

IP: 35.173 .219 .12

26 апреля 2023 г., 13:17:19

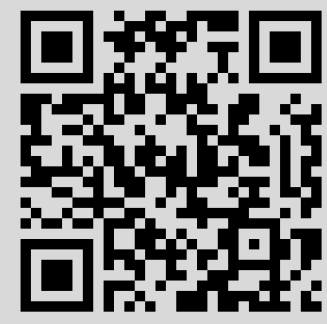




\section{Об одном обобщении сумм Клостермана}

\section{А. В. Устинов}

Для целых $u, v$ и натурального $q$ суммы Клостермана определяются равенством

$$
K_{q}(u, v)=\sum_{z=1}^{q} e\left(\frac{u z+v z^{-1}}{q}\right),
$$

где $e(x)=e^{2 \pi i x}$, и знак звездочки означает, что суммирование проходит по приведенной системе вычетов по модулю $q$.

Используя оценку Вейля (см. [1])

$$
\left|K_{p}(u, v)\right| \leqslant 2 p^{1 / 2},
$$

где $p$ - простое число, $p \nmid(u, v)$, Эстерман доказал (см. [2]), что для произвольного натурального $q$

$$
\left|K_{q}(u, v)\right| \leqslant \tau(q)(u, v, q)^{1 / 2} q^{1 / 2}
$$

$(\tau(q)$ - число делителей $q)$. Эта оценка применяется во многих теоретико-числовых задачах, см. обзор [2]. В связи с изучением распределения решений уравнения $\operatorname{det} X=N$, где $N$ - растущее натуральное число, а $X$ - матрица $3 \times 3$ с целыми коэффициентами, возникает необходимость рассматривать суммы более общего вида (см. [1])

$$
K_{q, d}(u, v ; w)=\sum_{\substack{z=1 \\ z \equiv w(\bmod d)}}^{q} e\left(\frac{u z+v z^{-1}}{q}\right),
$$

где $d$ - натуральное число и $d \mid q$. Сумма $K_{q, d}(u, v ; w)$ совпадает с суммой Клостермана $K_{q}(u, v)$ при $d=1$.

Метод доказательства оценки (1), предложенный в статье [2], можно применить и для оценки сумм (2). Всегда будем предполагать, что $w \in \mathbb{Z}_{d}^{*}$, поскольку в противном случае сумма $K_{q, d}(u, v ; w)$ пуста. Через $\delta_{q}(n)$ будем обозначать характеристическую функцию делимости на $q$ :

$$
\delta_{q}(n)= \begin{cases}1, & \text { если } n \equiv 0(\bmod q), \\ 0, & \text { если } n \neq \equiv 0(\bmod q) .\end{cases}
$$

Teоpema 1. Пусть $D=(d, q / d) u w \in \mathbb{Z}_{d}^{*}$. Тогдa

$$
\left|K_{q, d}(u, v ; w)\right| \leqslant \delta_{D}\left(u-v w^{-2}\right) \tau(q)(u, v, q)^{1 / 2} q^{1 / 2} .
$$

Доказательству теоремы предпошлем несколько вспомогательных утверждений.

ЗАмечАниЕ 1. Суммы Клостермана обладают свойством мультипликативности по $q$. Если $q=q_{1} q_{2},\left(q_{1}, q_{2}\right)=1$, то с помощью замены $z=z_{1} q_{2}+z_{2} q_{1}, 1 \leqslant z_{1} \leqslant q_{1}, 1 \leqslant z_{2} \leqslant q_{2}$, проверяется равенство

$$
K_{q}(u, v)=K_{q_{1}}\left(u, v_{1}\right) K_{q_{2}}\left(u, v_{2}\right),
$$

где $v=v_{1} q_{2}^{2}+v_{2} q_{1}^{2}$ (см. [3; 55.6$\left.]\right)$. Аналогично при тех же предположениях проверяется и мультипликативное свойство сумм (2):

$$
K_{q, d}(u, v ; w)=K_{q_{1}, d_{1}}\left(u, v_{1} ; w_{1}\right) K_{q_{2}, d_{2}}\left(u, v_{2} ; w_{2}\right),
$$

Работа выполнена при поддержке Российского фонда фундаментальных исследований (грант № 14-01-900025 Бел_а) и фонда "Династия".

DOI: $10.4213 / \mathrm{mzm} 10575$

(C) А. В. Устинов, 2015 
где

$$
d_{1}=\left(d, q_{1}\right), \quad d_{2}=\left(d, q_{2}\right), \quad w_{1} \equiv w q_{2}^{-1}\left(\bmod d_{1}\right), \quad w_{2} \equiv w q_{1}^{-1}\left(\bmod d_{2}\right) .
$$

Поскольку обе части неравенства (3) являются мультипликативными функциями от $q$, теорему 1 достаточно проверить в случае, когда $q$ есть степень простого числа.

В частности, из равенства (4) следует, что

$$
K_{q, d}(0,0 ; w)= \begin{cases}\frac{\varphi(q)}{\varphi(d)}, & \text { если }(w, d)=1 ; \\ 0 & \text { иначе. }\end{cases}
$$

ЗАмечАниЕ 2. Если $p$ - простое число, $q=p^{m}, d=p^{\alpha}(\alpha \leqslant m)$, то при $\alpha=0$ оценка (3) совпадает с неравенством (1), поэтому достаточно ограничиться рассмотрением случая $1 \leqslant$ $\alpha \leqslant m$

Лемма 1. Пусть $p-$ простое число $u(w, p)=1$. Тогда

$$
\begin{array}{lll}
\left(w+p^{n} t\right)^{-1} \equiv w^{-1}-p^{n} t w^{-2}\left(\bmod p^{m}\right), & m \leqslant 2 n, \\
\left(w+p^{n} t\right)^{-1} \equiv w^{-1}-p^{n} t w^{-2}+p^{2 n} t^{2} w^{-3}\left(\bmod p^{m}\right), & & m \leqslant 3 n .
\end{array}
$$

В лемме 1 и далее предполагается, что обратные элементы вычисляются по модулю, равному максимальной степени простого числа, входящей в сравнение.

ДоказАтельство. См. леммы 1 и 5 в [2].

Лемма 2. Пусть $p>2$ - простое число. Тогда

$$
\left|\sum_{t=1}^{p^{n+1}} e\left(\frac{a t^{2}}{p}+\frac{h t}{p^{n+1}}\right)\right|= \begin{cases}p^{n+1 / 2}, & \text { если } p^{n} \mid h, p \nmid a ; \\ 0, & \text { если } p^{n} \nmid h .\end{cases}
$$

ДоказАтельство. См. лемму 7 в [2].

Лемма 3. Пусть $p-$ простое число, $q=p^{m}, d=p^{\alpha},(w, p)=1 u 1 \leqslant \alpha \leqslant m \leqslant 2 \alpha$. Тогда

$$
K_{q, d}(u, v ; w)=e\left(\frac{u w+v w^{-1}}{p^{m}}\right) p^{m-\alpha} \delta_{p^{m-\alpha}}\left(u-v w^{-2}\right) .
$$

ДокАзАтЕльство. Для проверки утверждения леммы достаточно сделать замену переменной $z=w+p^{\alpha} z_{1}\left(1 \leqslant z_{1} \leqslant p^{m-\alpha}\right)$ и воспользоваться сравнением (5):

$K_{q, d}(u, v ; w)=\sum_{z_{1}=1}^{p^{m-\alpha}} e\left(\frac{u w+v w^{-1}}{p^{m}}+\frac{z_{1}\left(u-v w^{-2}\right)}{p^{m-\alpha}}\right)=e\left(\frac{u w+v z^{-1}}{p^{m}}\right) p^{m-\alpha} \delta_{p^{m-\alpha}}\left(u-v w^{-2}\right)$.

Лемма 4. Если $p$ - простое число, $q=p^{m}, d=p^{\alpha}, 1 \leqslant \alpha \leqslant m-n, \alpha \leqslant m / 2 \leqslant n \leqslant m$, $p \nmid(u, v),(p, w)=1$. Тогда

$$
\left|K_{q, d}(u, v ; w)\right| \leqslant A p^{n} \delta_{p^{\alpha}}\left(u-v w^{-2}\right),
$$

где $A=4$, если $p=2, m-n \geqslant \alpha+2$ и $A=2$ в остальных случаях.

Доказательство. Сделаем замену переменной суммирования: $z=s+p^{n} t, 1 \leqslant s \leqslant p^{n}$, $1 \leqslant t \leqslant p^{m-n}, s \equiv w\left(\bmod p^{\alpha}\right)$. Тогда

$$
\begin{aligned}
K_{q, d}(u, v ; w) & =\sum_{\substack{s=1 \\
s \equiv w\left(\bmod p^{\alpha}\right)}}^{p^{n}} e\left(\frac{u s+v s^{-1}}{p^{m}}\right) \sum_{t=1}^{p^{m-n}}\left(\frac{t\left(u-v s^{-2}\right)}{p^{m-n}}\right) \\
& =p^{m-n} \sum_{\substack{s=1 \\
s \equiv w\left(\bmod p^{\alpha}\right)}}^{p^{n}} e\left(\frac{u s+v s^{-1}}{p^{m}}\right) \delta_{p^{m-n}}\left(u-v s^{-2}\right) .
\end{aligned}
$$

По условию $m-n \geqslant \alpha$. Поэтому сумма будет ненулевой только если $\delta_{p^{\alpha}}\left(u-v w^{-2}\right)=1$. В этом случае при $p>2$ решение сравнения $u-v s^{-2} \equiv 0\left(\bmod p^{\alpha}\right)$ (относительно неизвест- 
ной $s$ ) однозначно поднимается до решения по модулю $p^{m-n}$, т.е. сумма по переменной $s$ состоит не более чем из $p^{2 n-m}$ слагаемых, и

$$
\left|K_{q, d}(u, v ; w)\right| \leqslant p^{n} \delta_{p^{\alpha}}\left(u-v w^{-2}\right) .
$$

Если же $p=2$, то сравнение $u-v s^{-2} \equiv 0\left(\bmod p^{m-n}\right)$ при $m-n=\alpha+1$ имеет не более 2 решений, а при $m-n \geqslant \alpha+2-$ не более 4 решений. Таким образом сумма по переменной $s$ состоит не более чем из $A p^{2 n-m}$ слагаемых, и

$$
\left|K_{q, d}(u, v ; w)\right| \leqslant A p^{n} \delta_{p^{\alpha}}\left(u-v w^{-2}\right),
$$

где $A=4$, если $m-n \geqslant \alpha+2, A=2$, если $m-n=\alpha+1$ и $A=1$, если $m-n=\alpha$.

Лемма 5. Пусть $d=2^{\alpha}, \alpha \geqslant 1, m \geqslant 2 \alpha, 2 \nmid(u, v) u(2, w)=1$. Тогда

$$
\left|K_{2^{m}, d}(u, v ; w)\right| \leqslant \tau\left(2^{m}\right) 2^{m / 2} \delta_{2^{\alpha}}\left(u-v w^{-2}\right) .
$$

ДокАЗАтЕЛЬСтво. Применим лемму 4 с $n=\lceil m / 2\rceil$. Тогда при четном $m=2 n$

$$
\left|K_{2^{m}, d}(u, v ; w)\right| \leqslant A 2^{m / 2} \delta_{2^{\alpha}}\left(u-v w^{-2}\right) \leqslant \tau\left(2^{m}\right) 2^{m / 2} \delta_{2^{\alpha}}\left(u-v w^{-2}\right),
$$

где $A=4$ при $n \geqslant \alpha+2$ и $A=2$ в остальных случаях. Если же $m$ - нечетное, то $m=$ $2 n-1$ и

$$
\left|K_{2^{m}, d}(u, v ; w)\right| \leqslant A 2^{(m+1) / 2} \delta_{2^{\alpha}}\left(u-v w^{-2}\right) \leqslant \tau\left(2^{m}\right) 2^{m / 2} \delta_{2^{\alpha}}\left(u-v w^{-2}\right) .
$$

Чтобы убедиться в последнем неравенстве, достаточно проверить, что $\sqrt{2} A \leqslant \tau\left(2^{m}\right)=m+1$. Если $A=4$, то $m-n \geqslant 3, m \geqslant 7$, и неравенство выполняется, поскольку $4 \sqrt{2} \leqslant 8$. Если же $A=2$, то неравенство $\sqrt{2} A \leqslant m+1$ выполняется для всех нечетных $m \geqslant 2 \alpha \geqslant 2$.

Лемма 6. Пусть $p>2$ - простое число, $d=p^{\alpha}, p \nmid(u, v),(p, w)=1 u m \geqslant 2 \alpha$. Тогда

$$
\left|K_{p^{m}, d}(u, v ; w)\right| \leqslant 2 p^{m / 2} \delta_{p^{\alpha}}\left(u-v w^{-2}\right) .
$$

ДокАЗАтЕльство. Если $m$ - четное число, то требуемая оценка следует из леммы 4 при $n=m / 2$. При нечетном $m$ положим $n=(m-1) / 2$, сделаем замену переменной $z=$ $s+p^{n} t, 1 \leqslant s \leqslant p^{n}, 1 \leqslant t \leqslant p^{n+1}$, и применим формулу (6):

$$
K_{p^{m}, d}(u, v ; w)=\sum_{\substack{s=1 \\ s \equiv w\left(\bmod p^{\alpha}\right)}}^{p^{n}} e\left(\frac{u s+v s^{-1}}{p^{m}}\right) \sum_{t=1}^{p^{n+1}}\left(\frac{t\left(u-v s^{-2}\right)}{p^{n+1}}+\frac{v t^{2} s^{-3}}{p}\right) .
$$

По лемме 2

$$
\left|K_{p^{m}, d}(u, v ; w)\right| \leqslant \sum_{\substack{s=1 \\ s \equiv w\left(\bmod p^{\alpha}\right)}}^{p^{n}} p^{n+1 / 2} \delta_{p^{n}}\left(u-v s^{-2}\right)=p^{m / 2} \sum_{\substack{s=1 \\ s \equiv w\left(\bmod p^{\alpha}\right)}}^{p^{n}} \delta_{p^{n}}\left(u-v s^{-2}\right),
$$

откуда и следует нужное неравенство, поскольку сумма по переменной $s$ (когда она не пуста) не превосходит двух.

ДокАЗАтЕльство теоремы 1. Согласно замечаниям 1-2 утверждение теоремы 1 достаточно проверить в случае, когда $q=p^{m}, d=p^{\alpha}$, где $p$ простое и $1 \leqslant \alpha \leqslant m$. При $\alpha \leqslant m \leqslant 2 \alpha$ оценка (3) следует из леммы 3 . Поэтому можно предполагать, что $m>2 \alpha$. Если $(u, v, q)=1$, то требуемая оценка следует из лемм 5 и 6 . Таким образом, остается рассмотреть случай, когда $(u, v, q)=p^{h}, 0<h \leqslant m$. При $h=m$ доказываемая оценка хуже тривиальной, поэтому будем считать, что $h<m$. Если $m-h \leqslant 2 \alpha$, то сумму достаточно оценить тривиально:

$$
\begin{gathered}
K_{q, d}(u, v ; w)=\sum_{\substack{z=1 \\
z \equiv w(\bmod d)}}^{p^{m}} e\left(\frac{u z+v z^{-1}}{p^{m}}\right)=\sum_{\substack{z=1 \\
z \equiv w(\bmod d)}}^{p^{m}} e\left(\frac{u p^{-h} z+v p^{-h} z^{-1}}{p^{m-h}}\right), \\
\left|K_{q, d}(u, v ; w)\right| \leqslant p^{m-\alpha} \leqslant \delta_{D}\left(u-v w^{-2}\right)(u, v, q)^{1 / 2} q^{1 / 2}=\delta_{D}\left(u-v w^{-2}\right) p^{(m+h) / 2} .
\end{gathered}
$$


Если же $m-h>2 \alpha$, то

$$
K_{q, d}(u, v ; w)=p^{h} \sum_{\substack{z=1 \\ z \equiv w(\bmod d}}^{p^{m-h}} e\left(\frac{u p^{-h} z+v p^{-h} z^{-1}}{p^{m-h}}\right)=p^{h} K_{q p^{-h}, d}\left(u p^{-h}, v p^{-h} ; w\right) .
$$

Снова применяя леммы 5 и 6 , приходим к утверждению теоремы:

$$
\begin{aligned}
\left|K_{q, d}(u, v ; w)\right| & \leqslant p^{h}\left|K_{q p^{-h}, d}\left(u p^{-h}, v p^{-h} ; w\right)\right| \\
& \leqslant \tau\left(q p^{-h}\right) p^{h} \delta_{p^{\alpha}}\left(u p^{-h}-v p^{-h} w^{-2}\right)\left(u p^{-h}, v p^{-h}, q p^{-h}\right)^{1 / 2}\left(q p^{-h}\right)^{1 / 2} \\
& \leqslant \tau(q) \delta_{p^{\alpha+h}}\left(u-v w^{-2}\right)(u, v, q)^{1 / 2} q^{1 / 2} \leqslant \tau(q) \delta_{D}\left(u-v w^{-2}\right)(u, v, q)^{1 / 2} q^{1 / 2} .
\end{aligned}
$$

Суммы Рамануджана

$$
c_{q}(u)=\sum_{z=1}^{q} e\left(\frac{u z}{q}\right)
$$

являются частными случаями сумм Клостермана: $K_{q}(u, 0)=K_{q}(0, u)=c_{q}(u)$, и допускают более точную оценку (см. [3; теорема 272])

$$
\left|c_{q}(n)\right| \leqslant(q, n)
$$

Если один из аргументов $u, v$ суммы $K_{q, d}(u, v ; w)$ равен нулю, то оценку теоремы 1 также можно уточнить.

Теорема 2. Справедливы неравенства

$$
\left|K_{q, d}(u, 0 ; w)\right| \leqslant\left(u, \frac{q}{d}\right), \quad\left|K_{q, d}(0, v ; w)\right| \leqslant\left(v, \frac{q}{d}\right) .
$$

ДоказАтельство. Из равенства $K_{q, d}(u, v ; w)=K_{q, d}\left(v, u ; w^{-1}\right)$ следует, что для доказательства теоремы достаточно проверить первое из неравенств (8). По свойству мультипликативности (см. замечание 1) неравенство (8) достаточно проверить для случая, когда $q$ есть степень простого числа. Предположим, что $q=p^{m}, d=p^{\alpha}$, где $p$ - простое число. Если $\alpha=0$, то утверждение теоремы следует из оценки (7):

$$
\left|K_{q, d}(u, 0 ; w)\right|=\left|\sum_{z=1}^{q} e\left(\frac{u z}{q}\right)\right|=\left|c_{q}(u)\right| \leqslant(u, q)=\left(u, \frac{q}{d}\right) .
$$

Если же $\alpha>0$, то

$$
\begin{aligned}
K_{q, d}(u, 0 ; w) & =\sum_{\substack{z=1 \\
z \equiv w(\bmod d)}}^{q} e\left(\frac{u z}{q}\right)=\sum_{z_{1}=1}^{p^{m-\alpha}} e\left(\frac{u\left(w+p^{\alpha} z_{1}\right)}{q}\right), \\
\left|K_{q, d}(u, 0 ; w)\right| & =p^{m-\alpha} \delta_{p^{m-\alpha}}(u) \leqslant\left(u, \frac{q}{d}\right) .
\end{aligned}
$$

Автор благодарит М. А. Королева за указания на недостатки в первоначальной версии статьи.

\section{СПИСОК ЦИТИРОВАННОЙ ЛИТЕРАТУРЫ}

[1] Ю. В. Линник, Б. Ф. Скубенко, Вестн. ЛГУ. Сер. матем., мех., астрон., 3:13 (1964), 25-36. [2] T. Estermann, Mathematika, 8 (1961), 83-86. [3] G. H. Hardy, E. M. Write, An Introduction to the Theory of Numbers, Clarendon Press, Oxford, 1979. [4] I. E. Shparlinski, Jpn. J. Math., 7:2 (2012), 235-294. [5] A. Weil, Proc. Natl. Acad. Sci. USA, 34:5 (1948), 204-207.

\section{А. В. Устинов}

Хабаровское отделение

Института прикладной математики ДВО РАН

E-mail: ustinov.alexey@gmail.com
Поступило

24.02 .2014

Исправленный вариант

14.05.2014 